\title{
Corneal collagen cross-linking epithelium-on vs. epithelium-off: a systematic review and meta-analysis
}

Francesco D'Oria ${ }^{1,2,3}$, Antonio Palazón ${ }^{4}$ and Jorge L. Alio ${ }^{1,2^{*}}$

\begin{abstract}
Background: The purpose of the study was to determine the advantages and disadvantages of epi-on corneal crosslinking (CXL) techniques compared with standard epi-off CXL.

Methods: We searched MEDLINE and EMBASE for randomized controlled trials (RCTs) and non-randomized studies of interventions (NRSIs) and we evaluated the selected papers according to the Cochrane risk of bias tool. We considered, as primary outcomes, average Kmax flattening, changes in uncorrected and corrected distance visual acuity (UDVA and CDVA); as secondary outcomes, we considered changes in pachymetry values and endothelial cell density (ECD). We also investigated adverse events related to the treatments and treatment failure. Meta-analysis was conducted with a fixed or random-effects model using weighted mean difference (MD) with 95\% confidence interval (Cl) as the effect size.
\end{abstract}

Results: A total of 15 studies were included and among these 15 trials, 9 were RCTs and 6 were NRSIs, but only 4 studies showed no high risk of bias and were included in this meta-analysis. Our analysis revealed significant postoperative differences in CDVA (MD $=0.07 ; 95 \% \mathrm{Cl} 0.04$ to $0.10 ; P<0.001)$, and no significative differences in UDVA, Kmax, central corneal thickness (CCT) and ECD $(P>0.05)$. Epi-on CXL protocol was found to be significantly less prompt to have risks of delay in epithelial healing $(P=0.035)$ and persistent stromal haze $(P=0.026)$.

Conclusion: Epi-on CXL is as effective as epi-off CXL. Except for a higher significant improvement in CDVA with current epi-on protocols, our meta-analysis demonstrates that epi-on and epi-off CXL have comparable effects on visual, topographic, pachymetric, and endothelial parameters. Epi-on CXL has clinical advantages in terms of comfort and avoidance of complications as it reduces the risk of developing delay in epithelial healing and persistent stromal haze.

Keywords: Corneal collagen cross-linking, Keratoconus, Transepithelial CXL, Epithelium-off CXL, Epithelium-on CXL, lontophoresis

\section{Background}

Keratoconus is an ectatic corneal disorder affecting up to 1:375 in some populations, characterized by a progressive deformation and thinning of the cornea [1]. Disease detection is essential for improving the management of keratoconus patients as it can advance from mild changes

*Correspondence: jlalio@vissum.com

1 Vissum Innovation, c/ Cabañal, 1, 03016 Alicante, Spain

Full list of author information is available at the end of the article to a severe loss of visual acuity that might require surgical approaches [2]. Several classification systems have been proposed to grade keratoconus as older ones e.g., Amsler-Krumeich classification system [3], although widely accepted, do not consider other variables such as the anterior corneal higher-order aberration (HOA) $[4$, 5].

Corneal cross-linking (CXL) was introduced in the late 1990s as a therapeutic approach to strengthen the original author(s) and the source, provide a link to the Creative Commons licence, and indicate if changes were made. The images or other third party material in this article are included in the article's Creative Commons licence, unless indicated otherwise in a credit line to the material. If material is not included in the article's Creative Commons licence and your intended use is not permitted by statutory regulation or exceeds the permitted use, you will need to obtain permission directly from the copyright holder. To view a copy of this licence, visit http://creativecommons.org/licenses/by/4.0/. The Creative Commons Public Domain Dedication waiver (http://creativeco mmons.org/publicdomain/zero/1.0/) applies to the data made available in this article, unless otherwise stated in a credit line to the data. 
biomechanical and biochemical properties of the cornea and the first clinical results were published by Wallensak et al. in 2003 [6]. The original CXL procedure is known as the "Dresden protocol" and is an epithelium-off procedure. In the standard technique, after anesthetizing the eye, the central $8 \mathrm{~mm}$ of the corneal epithelium is removed to expose the collagen-rich stroma and riboflavin solution (0.1\% riboflavin-5-phosphate and $20 \%$ dextran T-500) is applied to the surface of the cornea both $30 \mathrm{~min}$ before irradiation and at $5 \mathrm{~min}$ intervals during a $30 \mathrm{~min}$ exposure to $370 \mathrm{~nm}$ UVA with a fluence of $3 \mathrm{~mW} /$ $\mathrm{cm}^{2}$ and a total irradiation dose of $5.4 \mathrm{~J} / \mathrm{cm}^{2}$ [7]. The downside of epithelial removal is that it causes significant pain and discomfort in the early postoperative period; epi-off CXL carries a small risk of viral reactivation, haze, melting, infectious ulceration and the development of permanent stromal scars [8].

Considering these situations, several variations of the standard CXL procedure have been proposed since its introduction. Transepithelial or epi-on CXL is one such variation. Leaving the corneal epithelium intact should reduce pain and complications associated with epithelial debridement, such as infectious keratitis and could also lead to a shorter interruption of contact lens wear [8]. Considering transepithelial procedures, it should be considered that riboflavin is a large hydrophilic molecule that cannot penetrate an intact epithelium; moreover, the epithelium blocks about $20 \%$ of the UV illumination administered [9]. To improve riboflavin penetration into the stroma via the intact epithelium, several approaches have been used and investigated to encourage riboflavin penetration to the stroma [9].

There is, however, a paucity of studies that have been constructed to answer the clinical question of relative benefit for these procedures, and thus the evidence available for evaluation is limited. The main objectives of this systematic review and meta-analysis were to determine, in an evidence-based manner and using the currently available literature, the advantages and disadvantages of epi-on CXL techniques compared with traditional epi-off CXL and to discuss their indications.

\section{Methods}

The review followed methods recommended by Cochrane and the Centre for Reviews and Dissemination [10] and is reported according to PRISMA guidelines [11]. Furthermore, it has been registered in the PROSPERO database (CRD42020156072), where the review methods were established prior to the beginning of the review analysis.

\section{Method of literature search}

Two reviewers (FD and JLA) independently performed a systematic literature search in the MEDLINE/PubMed database and the EMBASE database from January 2014 to July 2021. This range was chosen to ensure that only contemporary and comparable procedures for CXL were included. The following keywords were used: "Keratoconus", "Cross-Linking Reagents", "Iontophoresis", "Riboflavin", "Epithelium", "Ultraviolet Rays", "Epi-On", "Epi-Off", "Epithelium-On", "Epithelium-Off". These were searched in the title and the abstract as well as MESH/ EMTREE terms. Search terms are shown in Additional file 2. Only articles published in English, Italian, or Spanish with an available abstract were included in the systematic literature search (languages in which the authors had good command). The abstracts of related titles were reviewed, and the full articles retrieved if their title or abstract appeared to meet the objectives of this review. Studies were included if they met the inclusion criteria. Reference lists of the included papers were screened too. Moreover, we manually analyzed the following trial registries: clinicaltrials.gov and Cochrane Controlled Register of Trials as well as "grey literature".

\section{Study selection, inclusion and exclusion criteria}

All publications were screened by two authors (FD and JLA) and any disagreement was discussed by the two authors and resolved by consensus. The reports were screened according to the following selection criteria: (a) studies that compared the role of epi-on CXL to epioff CXL for progressive keratoconus patients; (b) clinical trial studies; (c) both randomized controlled trials (RCTs) and non-randomized studies of interventions (NRSI), to generate evidence that will guide medical decisions [12]. All the studies that we reviewed were classified based on their scientific level of evidence according to the General Guidelines for Methodologies on Research and Evaluation of Traditional Medicine of the World Health Organization [13]. Only articles with a level Ib or IIa scientific evidence were selected. All the articles that were found were carefully reviewed to select those that reported original clinical data pre- and postoperatively. Data from previously reported cases included in different articles were omitted to avoid duplication of data. Articles on corneal collagen cross-linking combined with other treatments, such as topography-guided photorefractive keratectomy or intrastromal corneal ring segments were excluded. Experimental animal studies and ex vivo investigational reports were excluded from the review analysis. We also excluded all studies with a follow-up period of less than 12 months. 


\section{Quality assessment}

Two reviewers (FD and JLA) separately evaluated the studies based on the methods recommended in the Risk of Bias in Non-randomized Studies of Intervention (ROBINS-I) [10, 14] for cohort studies based on seven domains (confounders, selection of participants into the study, classification of interventions, deviations from the intended intervention, missing data, measurement of outcomes, and selection of the reported results). The "Cochrane risk of bias tool" [12] was used to assess the methodological quality of RCTs, by examining the following domains: random sequence generation, allocation concealment, blinding of participants and personnel, blinding of outcome assessment, incomplete outcome data, selective reporting, and other biases. The review authors looked for sources of funding for the studies included in the review. Any disagreement was discussed by the reviewers and resolved.

\section{Data extraction and clinical outcome}

Two reviewers achieved consensus on which data to extract from the included studies (FD and JLA, followed the previous methods to achieve a consensus) and included the name of the first author, the year of publication, the trial location, the study design, the number of eyes, the type of CXL protocols used, the follow-up durations and outcome measures. In this review we considered, as the main outcome, average Kmax flattening, considering that the primary objective of CXL is to stabilize the underlying disease process, changes in uncorrected distance visual acuity (UDVA) and corrected distance visual acuity (CDVA), changes in HOAs and comatic aberrations, and depth of the demarcation line as an indirect treatment indicator of effectiveness. The secondary outcome parameters investigated in this study were changes in pachymetry values, either central corneal thickness $(\mathrm{CCT})$ or corneal thickness at the thinnest point and endothelial cell density (ECD). We also investigated adverse events related to the treatments and treatment failure, such as keratoconus progression, loss of $\geq 2$ lines of CDVA, delay in epithelial healing, persistent stromal haze, sterile infiltrates and infections.

\section{Statistical analysis}

If all the included clinical characteristics were similar between groups, we think that it would be reasonable to combine these studies through a meta-analysis. Otherwise, a descriptive analysis will be carried out. If we performed the meta-analysis with our extracted data, following the AMSTAR-2 (Assessment of Multiple Systematic Reviews) checklist [15], we will only combine those works with no high risk of bias. For continuous outcomes, the weighted mean difference (MD) will be calculated for obtaining the absolute changes. This calculation will be carried out through a random-effects models, unless there is significant heterogeneity or if we have less than three studies [16]. Heterogeneity across studies will be estimated by using Cochran's $\mathrm{Q}$ test and $\mathrm{I}^{2}$, considering its presence when $\mathrm{I}^{2}>50 \%$ and/or $P$-value $>0.10$.

If significant heterogeneity existed among trials, we would explore sources of heterogeneity, using subgroup analysis and meta-regression models, so long as we have enough studies [16]. We will perform sensitivity analysis by repeating the calculations excluding groups of studies (those with unclear risk of bias and which have used iontophoresis) each time and compare the results obtained. A group of studies was considered influential if it varied the overall coefficient by at least $10 \%$. Finally, we will analyze the asymmetry of funnel plots with the Egger test when we obtain at least 10 studies [17].

We set type I error at 5\% and for each relevant parameter, its associated confidence interval (CI) was calculated. The statistical software for this meta-analysis was R 3.3.3 through the meta for package (Meta-Analysis Package for R).

\section{Results}

\section{Systematic literature search}

This study identified 1102 publications after a systematic literature search and after the removal of duplicates as shown in the PRISMA flowchart (Fig. 1). Forty-four potentially relevant articles were identified and accessed in full text, 29 were excluded due to other design [18-30], level IIb or less of evidence [31-38], only abstract available [39-42], follow-up of less than 12 months [43, 44], same trial but with a shorter follow-up [45], and lack of data on comparison [46]. Fifteen relevant articles were included [47-61].

\section{Characteristics of included studies}

We identified a total of 15 studies where epi-on CXL outcomes of progressive keratoconus patients were compared to epi-off CXL outcomes. Among these 15 trials, 9 were RCTs $[48-50,54,55,57,59-61]$ and 6 were NRSIs [47, 51-53, 56, 58]. The country, study design, population and sample size, comparison, CXL regimen, outcome measures and follow-up of each clinical study were meticulously reviewed and summarized in Table 1. There were 511 eyes included in the standard epi-off CXL group and 574 eyes included in the epi-on CXL group. Studies were conducted in Italy, Jordan, Egypt, France, Peru, Netherlands, USA, Turkey, Russia and Saudi Arabia and were reported between 2015 and 2019. The duration of the follow-up ranged from 12 to 36 months. The study by Goodefroji et al. [54] derived data from a previously 

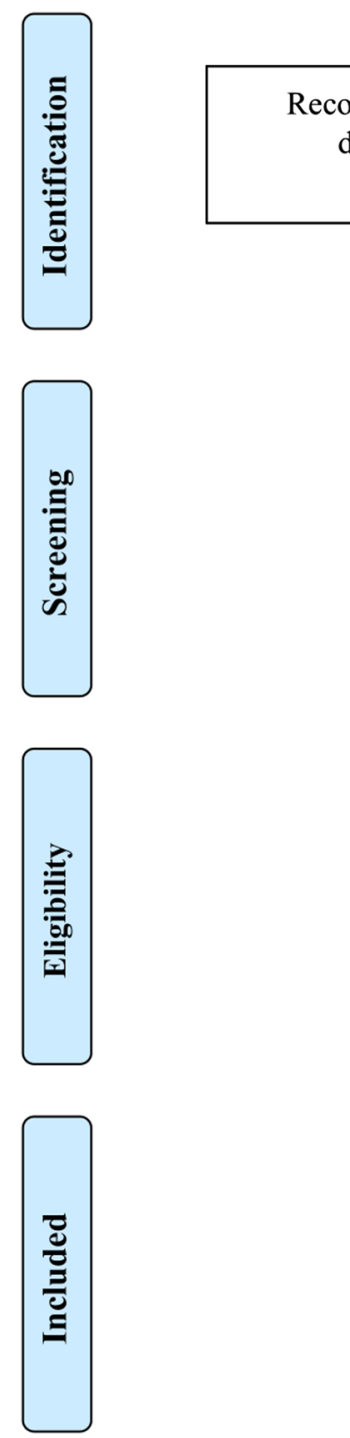

Records identified through
database searching $(\mathrm{n}=1,480)$
Additional records identified through other sources

$(\mathrm{n}=0)$

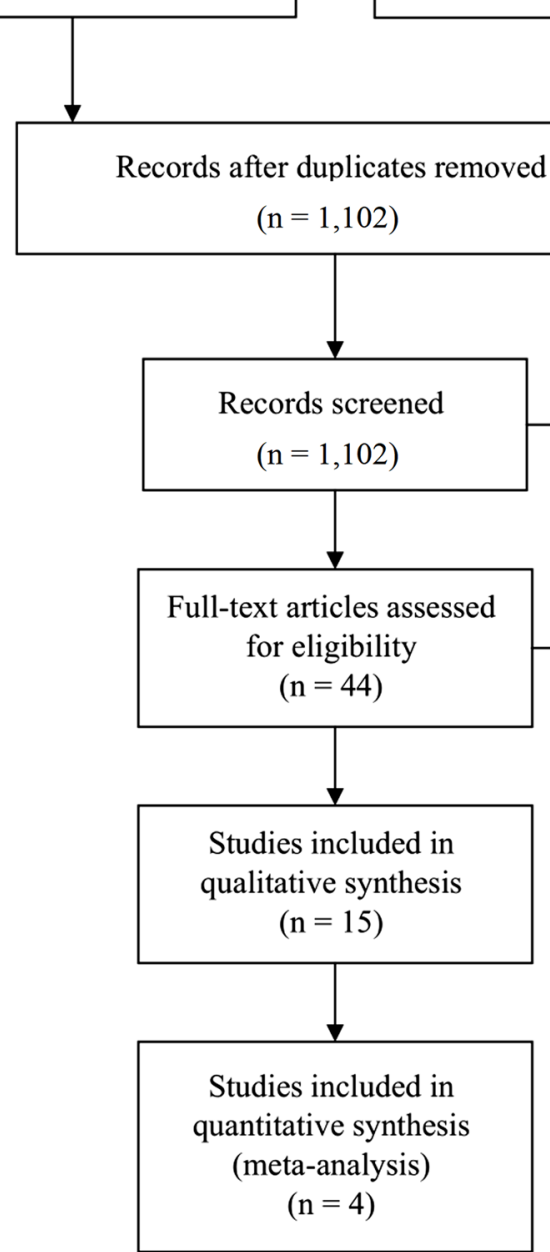

Records

excluded $(n=1,058)$

Full-text articles excluded

Other design $(\mathrm{n}=13)$

Low level of evidence $(n=8)$

Only abstract available $(n=4)$

Follow-up $<12$ months $(\mathrm{n}=2)$

Same trial $(n=1)$

Lack of data on comparison $(n=1)$

Fig. 1 Flow chart of eligible papers used in the meta-analysis (PRISMA statement). PRISMA, preferred reporting items for systematic reviews and meta-analyses

published RCT [61] to further investigate the development of HOAs and their effects on visual acuity, and thus only data concerning HOAs were extracted and included in the outcome measures (Tables 2, 3 and 4).

\section{Primary outcomes}

\section{Corneal keratometry}

Three studies compared the Kmax between the epi-on and epi-off CXL groups (Fig. 2) [48, 60, 61]. Among these studies (Fig. 2a), since there was evidence of heterogeneity $\left(\mathrm{Q}=4.2615, \mathrm{df}=2, P=0.1187 ; \mathrm{I}^{2}=54.28 \%\right)$, a random-effect model was used to calculate the pooled $\mathrm{MD}$ and its $95 \% \mathrm{CI}(\mathrm{MD}=-0.95 ; 95 \% \mathrm{CI}-2.31$, to
$0.42 ; P=0.174)$. The results of the sensitivity analysis (Table 4) showed a significant flattening of Kmax with epi-off CXL protocol considering only those with low risk of bias $(\mathrm{MD}=-1.69 ; 95 \% \mathrm{CI}-2.62$ to -0.76 ; $P=0.004)$.

\section{Visual acuity}

Three studies compared the UDVA (logMAR) between the epi-on and epi-off CXL-treated groups $[48,60,61]$. As there was no obvious heterogeneity $(\mathrm{Q}=0.3463$, $\left.\mathrm{df}=2, \quad P=0.8410 ; \mathrm{I}^{2}=0 \%\right)$, a fixed-effect model was applied to calculate the pooled MD and its 95\% CI $(\mathrm{MD}=-0.03 ; 95 \% \mathrm{CI}-0.09$ to $0.02 ; P=0.228)$ (Fig. $2 \mathrm{~b}$ ). 


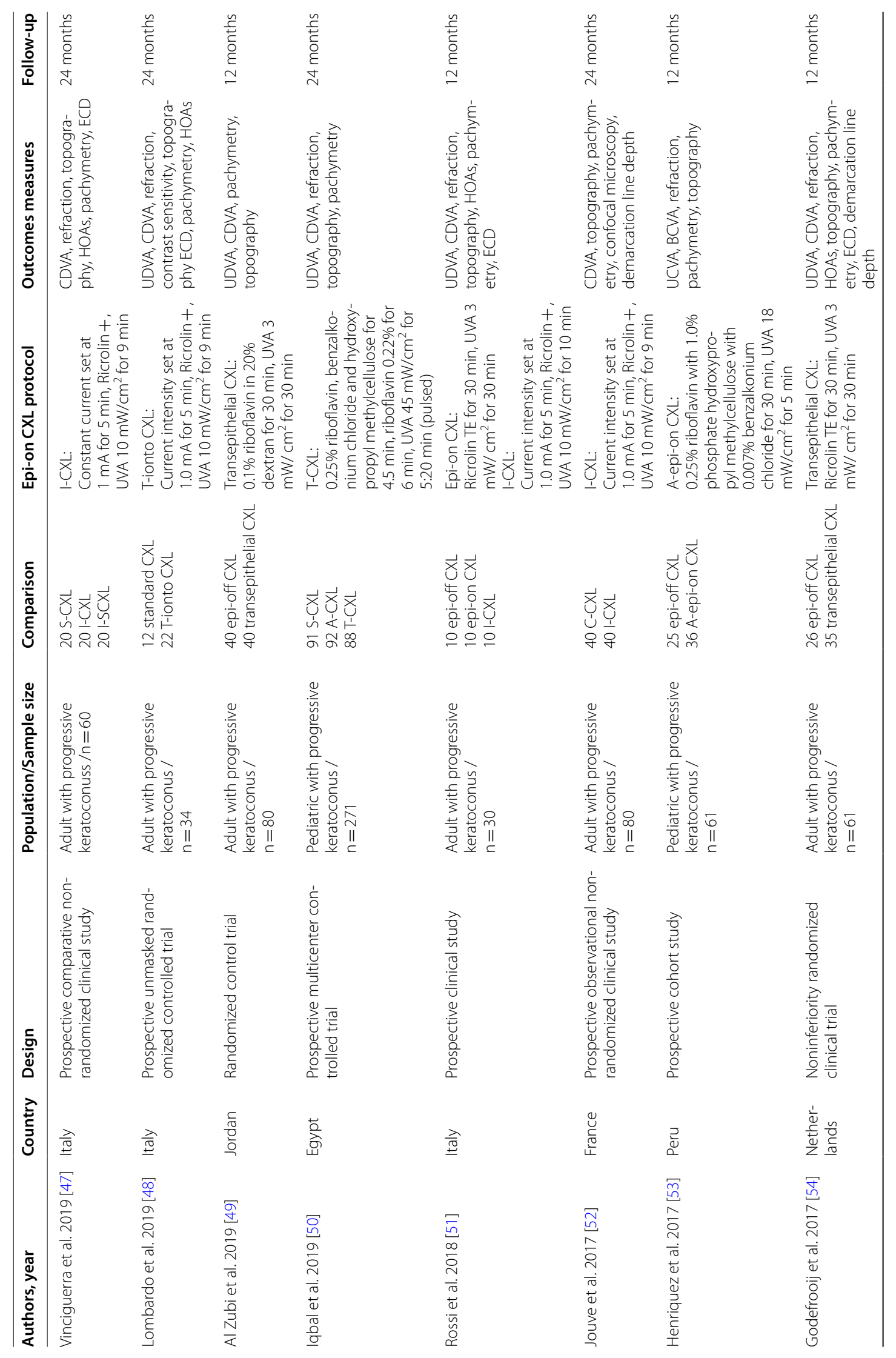




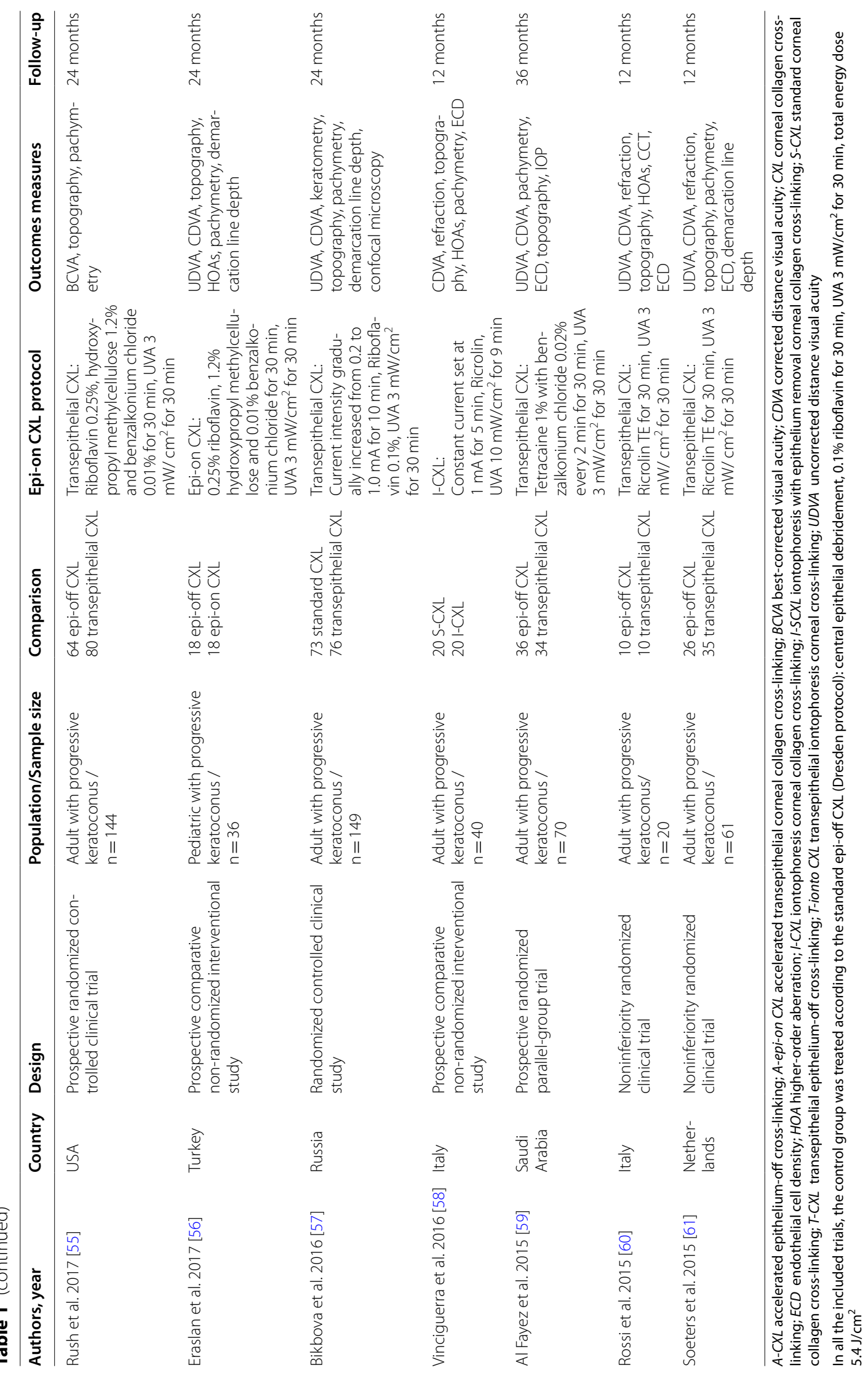


Table 2 Mean differences in the main outcomes and adverse events in randomized controlled trials after corneal collagen crosslinking (epi-on vs. epi-off)

\begin{tabular}{|c|c|c|c|c|c|c|}
\hline \multirow[t]{2}{*}{ Study or subgroup } & \multicolumn{3}{|l|}{ Epi-off CXL } & \multicolumn{3}{|l|}{ Epi-on CXL } \\
\hline & Mean \pm SD & n (\%) & $\mathrm{n}$ & Mean \pm SD & n (\%) & $\mathrm{n}$ \\
\hline \multicolumn{7}{|l|}{ Kmax (D) } \\
\hline Lombardo et al. 2019a [48] & $-1.5 \pm 4.9$ & - & 12 & $-1.0 \pm 3.7$ & - & 22 \\
\hline labal et al. 2019b [50] & $-1.2 \pm 1.0$ & - & 91 & $0.9 \pm 1.1$ & - & 88 \\
\hline 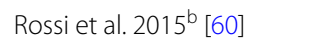 & $-1.1 \pm 2.1$ & - & 10 & $-1.1 \pm 1.0$ & - & 10 \\
\hline Soeters et al. $2015^{b}[61]$ & $-1.5 \pm 2.0$ & - & 26 & $0.3 \pm 1.8$ & - & 35 \\
\hline \multicolumn{7}{|l|}{ UDVA (logMAR) } \\
\hline Lombardo et al. 2019 [48] & $-0.3 \pm 0.3$ & - & 12 & $-0.3 \pm 0.4$ & - & 22 \\
\hline lqbal et al. 2019 [50] & $-0.3 \pm 0.1$ & - & 91 & $0.2 \pm 0.1$ & - & 88 \\
\hline Rossi et al. 2015 [60] & $-0.1 \pm 0.1$ & - & 10 & $-0.1 \pm 0.1$ & - & 10 \\
\hline Soeters et al. 2015 [61] & $-0.1 \pm 0.4$ & - & 26 & $-0.1 \pm 0.4$ & - & 35 \\
\hline \multicolumn{7}{|l|}{ CDVA (logMAR) } \\
\hline Lombardo et al. 2019 [48] & $-0.0 \pm 0.1$ & - & 12 & $-0.1 \pm 0.1$ & - & 22 \\
\hline Al Zubi et al. 2019 [49] & $-0.1 \pm 0.1$ & - & 40 & $-0.1 \pm 0.1$ & - & 40 \\
\hline lqbal et al. 2019 [50] & $-0.2 \pm 0.2$ & - & 91 & $0.1 \pm 0.1$ & - & 88 \\
\hline Rush et al. 2017 [55] & -0.2 & - & 64 & -0.1 & - & 80 \\
\hline Bikbova et al. 2016 [57] & $-0.0 \pm 0.3$ & - & 73 & $-0.1 \pm 0.5$ & - & 76 \\
\hline Rossi et al. 2015 [60] & $-0.1 \pm 0.0$ & - & 10 & $-0.2 \pm 0.1$ & - & 10 \\
\hline Soeters et al. 2015 [61] & $-0.1 \pm 0.2$ & - & 26 & $-0.1 \pm 0.21$ & - & 35 \\
\hline \multicolumn{7}{|l|}{ Higher-order aberrations ( $\mu \mathrm{m})$} \\
\hline Lombardo et al. 2019 [48] & $-0.1 \pm 0.9$ & - & 12 & $1.0 \pm 1.1$ & - & 22 \\
\hline Godefrooij et al. 2017 [54] & $-0.2 \pm 0.4$ & - & 26 & $0.0 \pm 0.5$ & - & 35 \\
\hline \multicolumn{7}{|l|}{ Comatic aberrations ( $\mu \mathrm{m})$} \\
\hline Rossi et al. 2015 [60] & $-0.4 \pm 1.2$ & - & 10 & $-0.7 \pm 1.3$ & - & 10 \\
\hline \multicolumn{7}{|c|}{ Demarcation Line (depth in $\mu \mathrm{m}$ at 1 month) } \\
\hline Bikbova et al. 2016 [57] & $287.0 \pm 15.0$ & - & 73 & $179.0 \pm 18.0$ & - & 76 \\
\hline Soeters et al. 2015 [61] & $266.0 \pm 64.0$ & - & 26 & 0.0 & - & 35 \\
\hline \multicolumn{7}{|c|}{ Central corneal thickness $(\mu \mathrm{m})$} \\
\hline Lombardo et al. 2019 [48] & $5.0 \pm 21.0$ & - & 12 & $7.0 \pm 27.0$ & - & 22 \\
\hline Al Zubi et al. 2019 [49] & $5.4 \pm 12.5$ & - & 40 & $6.7 \pm 15.5$ & - & 40 \\
\hline Bikbova et al. 2016 [57] & $-13.0 \pm 37.2$ & - & 73 & $-6.7 \pm 38.6$ & - & 76 \\
\hline Rossi et al. 2015 [60] & $1.8 \pm 14.6$ & - & 10 & $-2.7 \pm 37.3$ & - & 10 \\
\hline \multicolumn{7}{|l|}{ Corneal thinnest point $(\mu \mathrm{m})$} \\
\hline lqbal et al. 2019 [50] & $-8.9 \pm 14.9$ & - & 91 & $-6.7 \pm 9.0$ & - & 88 \\
\hline Soeters et al. 2015 [61] & $-4.0 \pm 8.0$ & - & 26 & $0.0 \pm 12.0$ & - & 35 \\
\hline \multicolumn{7}{|l|}{ ECD (cells $/ \mathrm{mm}^{2}$ ) } \\
\hline Lombardo et al. 2019 [48] & $-30.0 \pm 368.0$ & - & 12 & $-33.0 \pm 309.0$ & - & 22 \\
\hline Bikbova et al. 2016 [57] & $20.0 \pm 91.0$ & - & 73 & $17.0 \pm 69.0$ & - & 76 \\
\hline Rossi et al. 2015 [60] & $-31.4 \pm 66.6$ & - & 10 & $-53.0 \pm 202.4$ & - & 10 \\
\hline Soeters et al. 2015 [61] & $-59.0 \pm 284.2$ & - & 26 & $11.0 \pm 338.9$ & - & 35 \\
\hline \multicolumn{7}{|l|}{$\mathrm{KC}$ progression } \\
\hline Lombardo et al. 2019 [48] & - & $0(0.0 \%)$ & 12 & - & $2(9.1 \%)$ & 22 \\
\hline lqbal et al. 2019 [50] & - & $0(0.0 \%)$ & 91 & - & $25(28.4 \%)$ & 88 \\
\hline Bikbova et al. 2016 [57] & - & $0(0.0 \%)$ & 73 & - & $1(1.3 \%)$ & 76 \\
\hline Al Fayez et al. 2015 [59] & - & $0(0.0 \%)$ & 36 & - & $19(55.9 \%)$ & 34 \\
\hline Soeters et al. 2015 [61] & - & $0(0.0 \%)$ & 26 & - & $8(22.9 \%)$ & 35 \\
\hline \multicolumn{7}{|l|}{ Delay in epithelial healing } \\
\hline lqbal et al. 2019 [50] & - & $17(18.7 \%)$ & 91 & - & $0(0.0 \%)$ & 88 \\
\hline
\end{tabular}


Table 2 (continued)

\begin{tabular}{|c|c|c|c|c|c|c|}
\hline \multirow[t]{2}{*}{ Study or subgroup } & \multicolumn{3}{|l|}{ Epi-off CXL } & \multicolumn{3}{|l|}{ Epi-on CXL } \\
\hline & Mean \pm SD & n (\%) & $\mathrm{n}$ & Mean \pm SD & n (\%) & $\mathrm{n}$ \\
\hline Rush et al. 2017 [55] & - & $1(1.6 \%)$ & 64 & - & $0(0.0 \%)$ & 80 \\
\hline Bikbova et al. 2016 [57] & - & $4(5.5 \%)$ & 73 & - & $0(0.0 \%)$ & 76 \\
\hline Soeters et al. 2015 [61] & - & $2(7.7 \%)$ & 26 & - & $0(0.0 \%)$ & 35 \\
\hline \multicolumn{7}{|l|}{ Persistent stromal haze } \\
\hline Lombardo et al. 2019 [48] & - & $2(16.7 \%)$ & 12 & - & $0(0.0 \%)$ & 22 \\
\hline Al Zubi et al. 2019 [49] & - & $4(10.0 \%)$ & 40 & - & $0(0.0 \%)$ & 40 \\
\hline lqbal et al. 2019 [50] & - & $2(2.2 \%)$ & 91 & - & $0(0.0 \%)$ & 88 \\
\hline Bikbova et al. 2016 [57] & - & $4(5.5 \%)$ & 73 & - & $0(0.0 \%)$ & 76 \\
\hline Soeters et al. 2015 [61] & - & $1(3.8 \%)$ & 26 & - & $0(0.0 \%)$ & 35 \\
\hline \multicolumn{7}{|l|}{ Sterile infiltrates } \\
\hline Soeters et al. 2015 [61] & - & $1(3.8 \%)$ & 26 & - & $0(0.0 \%)$ & 35 \\
\hline \multicolumn{7}{|l|}{ Infection } \\
\hline Rush et al. 2017 [55] & - & $1(1.6 \%)$ & 64 & - & $0(0.0 \%)$ & 80 \\
\hline Soeters et al. 2015 [61] & - & $1(3.8 \%)$ & 26 & - & $0(0.0 \%)$ & 35 \\
\hline
\end{tabular}

CXL corneal collagen cross-linking; CDVA corrected distance visual acuity; $D$ diopter; $E C D$ endothelial cell density; $K C$ keratoconus; Kmax maximum keratometry; $\log M A R$ logarithm of the minimum angle of resolution; $n(\%)$ absolute frequency (relative frequency); $S D$ standard deviation; UDVA uncorrected distance visual acuity

${ }^{a}$ Combined Placido disk corneal topography and anterior segment optical coherence tomography (Visante Omni, Carl Zeiss Meditec AG)

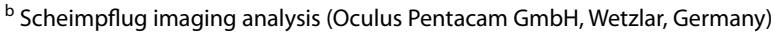

The exclusion of studies with unclear risk of bias or which have used iontophoresis from the full meta-analysis did not significantly alter the MD (Table 4 ), which ranged from -0.05 (95\% CI -0.20 to $0.10 ; P=0.491)$ to -0.01 (95\% CI -0.23 to $0.21 ; P<0.930$ ).

Four studies compared the CDVA (logMAR) between the epi-on and epi-off CXL-treated groups $[48,57,60,61]$. Since there was no obvious heterogeneity $(\mathrm{Q}=0.2896$, $\left.\mathrm{df}=3, P=0.1187 ; \mathrm{I}^{2}=0 \%\right)$, the random-effect model was used to calculate the pooled $\mathrm{MD}$ and its $95 \% \mathrm{CI}(\mathrm{MD}=0.07$; 95\% CI0.04 to $0.10 ; P<0.001$ ) (Fig. 2c). Sensitivity analysis showed that there was not a main source of heterogeneity. The results remained unchanged after removing the studies with an unclear risk of bias $(\mathrm{MD}=0.06 ; 95 \% \mathrm{CI}-0.004$ to $0.118 ; P=0.069)$ or with iontophoresis $(\mathrm{MD}=0.05 ; 95 \%$ $\mathrm{CI}-0.01$ to $0.11 ; P=0.127$ ).

\section{Secondary outcomes \\ Pachymetry}

Three studies compared the CCT between the epi-on and epi-off CXL groups (Fig. 2) [48, 57, 60]. There were no significant differences in CCT changes between the two groups $(\mathrm{MD}=3.52 ; 95 \% \mathrm{CI}-12.61$ to $5.58 ; P=0.448)$ and no heterogeneity between studies $(\mathrm{Q}=0.6306, \mathrm{df}=2$, $P=0.7296 ; \mathrm{I}^{2}=0 \%$ ) (Fig. $2 \mathrm{~d}$ ). The sensitivity analysis demonstrated that the result remained unchanged after the removal of the studies with an unclear risk of bias or performed with iontophoresis $(\mathrm{MD}=-2.0$ and -4.76 , respectively).

\section{Endothelial Cell Density}

Four studies compared the ECD between the epi-on and epi-off CXL-treated groups (Fig. 2) [48, 57, 60, 61]. There were no significant differences in ECD changes between the two groups $(\mathrm{MD}=1.80 ; 95 \% \mathrm{CI}-23.25$ to 26.86 ; $P=0.888$ ) (Fig. 2e). Sensitivity analysis demonstrated that the result remained unchanged after removal of the study by Lombardo et al. [48] ( $\mathrm{MD}=3.0 ; 95 \% \mathrm{CI}-22.86$ to 28.86; $P=0.468$ ), but the result did change after the removal of the studies with an unclear risk of bias $(\mathrm{MD}=-48.82 ; 95 \% \mathrm{CI}-180.79$ to $83.15 ; P=0.820)$. Potential publication bias for both the primary and secondary outcomes was not assessed due to the limited number of studies available for review.

\section{Adverse events and treatment failure}

Risk differences (RD) for adverse events and assessment of treatment failure in RCTs included in the quantitative analysis were assessed (Table 5). Epi-on CXL protocols were found to be significantly less prompt to have risks of delay in epithelial healing $(\mathrm{RD}=0.049 ; 95 \%$ CI0.003 to 0.0946; $P=0.035$ ) and persistent stromal haze ( $\mathrm{RD}=0.0525 ; 95 \% \mathrm{CI} 0.0063$ to $0.0986 ; P=0.026)$, and no significant for keratoconus progression $(\mathrm{RD}=-0.025$; 95\% CI -0.059 to $0.008 ; P=0.141$ ), sterile infiltrates $(\mathrm{RD}=0.0036 ; 95 \% \mathrm{CI}-0.0206$ to $0.0279 ; P=0.768)$ 
Table 3 Mean differences in the main outcomes and adverse events in non-randomized studies of interventions after corneal collagen cross-linking (epi-on vs. epi-off)

\begin{tabular}{|c|c|c|c|c|c|c|}
\hline \multirow[t]{2}{*}{ Study or subgroup } & \multicolumn{2}{|l|}{ Epi-off CXL } & \multirow[b]{2}{*}{$\mathbf{n}$} & \multicolumn{3}{|l|}{ Epi-on CXL } \\
\hline & Mean \pm SD & n (\%) & & Mean \pm SD & n (\%) & $\mathrm{n}$ \\
\hline \multicolumn{7}{|l|}{$\operatorname{Kmax}(\mathrm{D})$} \\
\hline Vinciguerra et al. 2019a [47] & $-0.1 \pm 4.6$ & - & 20 & $-0.4 \pm 4.4$ & - & 20 \\
\hline Jouve et al. 2017b [52] & $-1.1 \pm 4.2$ & - & 40 & $0.2 \pm 5.2$ & - & 40 \\
\hline Henriquez et al. 2017 [53] & $-0.9 \pm 5.2$ & - & 25 & $0.1 \pm 5.3$ & - & 36 \\
\hline Eraslan et al. 2017ª [55] & $-1.4 \pm 2.6$ & - & 18 & $-0.6 \pm 3.1$ & - & 18 \\
\hline Vinciguerra et al. 2016ª [57] & $-1.0 \pm 1.5$ & - & 20 & $-0.3 \pm 1.9$ & - & 20 \\
\hline \multicolumn{7}{|l|}{ UDVA (logMAR) } \\
\hline \multirow[t]{2}{*}{ Rossi et al. 2018 [51] } & $-0.1 \pm 0.2$ & - & 10 & $-0.2 \pm 0.2^{*}$ & - & $10^{*}$ \\
\hline & & & & $-0.1 \pm 0.2^{p}$ & - & $10^{\mathrm{p}}$ \\
\hline Henriquez et al. 2017 [53] & $-0.1 \pm 0.4$ & - & 25 & $-0.1 \pm 0.2$ & - & 36 \\
\hline Eraslan et al. 2017 [55] & $-0.0 \pm 0.1$ & - & 18 & $-0.1 \pm 0.2$ & - & 18 \\
\hline \multicolumn{7}{|l|}{ CDVA (logMAR) } \\
\hline Vinciguerra et al. 2019 [47] & $-0.1 \pm 0.1$ & - & 20 & $-0.1 \pm 0.2$ & - & 20 \\
\hline \multirow[t]{2}{*}{ Rossi et al. 2018 [51] } & $-0.1 \pm 0.1$ & - & 10 & $-0.1 \pm 0.0^{*}$ & - & $10^{*}$ \\
\hline & & & & $-0.1 \pm 0.1^{p}$ & - & $10^{\mathrm{p}}$ \\
\hline Jouve et al. 2017 [52] & $-0.1 \pm 0.1$ & - & 40 & $-0.1 \pm 0.2$ & - & 40 \\
\hline Henriquez et al. 2017 [53] & $-0.1 \pm 0.1$ & - & 25 & $-0.1 \pm 0.1$ & - & 36 \\
\hline Eraslan et al. 2017 [55] & $-0.1 \pm 0.1$ & - & 18 & $-0.1 \pm 0.1$ & - & 18 \\
\hline Vinciguerra et al. 2016 [57] & $-0.0 \pm 0.1$ & - & 20 & $-0.1 \pm 0.1$ & - & 20 \\
\hline \multicolumn{7}{|l|}{ Higher Order Aberrations ( $\mu \mathrm{m})$} \\
\hline Vinciguerra et al. 2019 [47] & $-0.1 \pm 0.3$ & - & 20 & $-0.7 \pm 0.2$ & - & 20 \\
\hline Vinciguerra et al. 2016 [57] & $-0.0 \pm 0.2$ & - & 20 & $-0.3 \pm 0.8$ & - & 20 \\
\hline \multicolumn{7}{|l|}{ Comatic Aberrations } \\
\hline Vinciguerra et al. 2019 [47] & $-0.3 \pm 1.0$ & - & 20 & $1.7 \pm 0.3$ & - & 20 \\
\hline \multirow[t]{2}{*}{ Rossi et al. 2018 [51] } & $-0.4 \pm 1.2$ & - & 10 & $-0.7 \pm 1.4^{*}$ & - & $10^{*}$ \\
\hline & & & & $-0.7 \pm 1.3^{p}$ & - & $10^{p}$ \\
\hline Eraslan et al. 2017 [55] & $-0.2 \pm 0.3$ & - & 18 & $-0.1 \pm 0.5$ & - & 18 \\
\hline Vinciguerra et al. 2016 [57] & $-0.2 \pm 0.3$ & - & 20 & $-1.2 \pm 1.6$ & - & 20 \\
\hline \multicolumn{7}{|c|}{ Demarcation Line (depth in $\mu \mathrm{m}$ at 1 month) } \\
\hline Jouve et al. 2017 [52] & $291.0 \pm 61.0$ & - & 40 & $216.0 \pm 49.0$ & - & 40 \\
\hline Eraslan et al. 2017 [55] & $272.3 \pm 28.6$ & - & 18 & $136.6 \pm 17.9$ & - & 18 \\
\hline \multicolumn{7}{|l|}{ Central corneal thickness $(\mu \mathrm{m})$} \\
\hline \multirow[t]{2}{*}{ Rossi et al. 2018 [51] } & $-2.9 \pm 18.9$ & - & 10 & $-1.6 \pm 35.5^{*}$ & - & $10^{*}$ \\
\hline & & & & $-4.7 \pm 30.2^{p}$ & - & $10^{p}$ \\
\hline \multicolumn{7}{|l|}{ Corneal Thinnest Point $(\mu \mathrm{m})$} \\
\hline Vinciguerra et al. 2019 [47] & $-57.0 \pm 103.0$ & - & 20 & $5.0 \pm 38.0$ & - & 20 \\
\hline Henriquez et al. 2017 [53] & $-12.5 \pm 40.2$ & - & 25 & $1.5 \pm 51.5$ & - & 36 \\
\hline Eraslan et al. 2017 [55] & $-11.3 \pm 14.9$ & - & 18 & $-8.8 \pm 14.8$ & - & 18 \\
\hline Vinciguerra et al. 2016 [57] & $-41.1 \pm 35.3$ & - & 20 & $1.0 \pm 7.2$ & - & 20 \\
\hline \multicolumn{7}{|l|}{ ECD (cells/mm²) } \\
\hline \multirow[t]{2}{*}{ Rossi et al. 2018 [51] } & $-32.7 \pm 99.3$ & - & 10 & $-46.1 \pm 197.9^{*}$ & - & $10^{*}$ \\
\hline & & & & $-27.0 \pm 62.5^{p}$ & - & $10^{p}$ \\
\hline \multicolumn{7}{|l|}{ KC progression } \\
\hline Jouve et al. 2017 [52] & - & $3(7.5 \%)$ & 40 & - & $8(20.0 \%)$ & 40 \\
\hline Henriquez et al. 2017 [53] & - & $3(12.0 \%)$ & 25 & - & $2(5.6 \%)$ & 36 \\
\hline \multicolumn{7}{|l|}{ Lost $\geq 2$ lines CDVA } \\
\hline Eraslan et al. 2017 [55] & - & $0(0.0 \%)$ & 18 & - & $1(5.6 \%)$ & 18 \\
\hline
\end{tabular}


Table 3 (continued)

\begin{tabular}{|c|c|c|c|c|c|c|}
\hline \multirow[t]{2}{*}{ Study or subgroup } & \multicolumn{2}{|l|}{ Epi-off CXL } & \multirow[b]{2}{*}{$\mathrm{n}$} & \multicolumn{3}{|l|}{ Epi-on CXL } \\
\hline & Mean \pm SD & n (\%) & & Mean \pm SD & n (\%) & $\mathbf{n}$ \\
\hline \multicolumn{7}{|l|}{ Persistent stromal haze } \\
\hline Henriquez et al. 2017 [53] & - & $1(0.1 \%)$ & 25 & - & $0(0.0 \%)$ & 36 \\
\hline Eraslan et al. 2017 [55] & - & $5(27.8 \%)$ & 18 & - & $0(0.0 \%)$ & 18 \\
\hline \multicolumn{7}{|l|}{ Sterile infiltrates } \\
\hline Henriquez et al. 2017 [53] & - & $1(0.1 \%)$ & 25 & - & $0(0.0 \%)$ & 36 \\
\hline
\end{tabular}

$C X L=$ corneal collagen cross-linking; $C D V A=$ corrected distance visual acuity; $D=$ diopter; $E C D$ endothelial cell density; $K C=$ keratoconus; $K m a x=$ maximum keratometry; $\log M A R=$ logarithm of the minimum angle of resolution; $n(\%)=$ absolute frequency (relative frequency); SD $=$ standard deviation; $U D V A=$ uncorrected distance visual acuity

*Transepithelial CXL treatment study group

${ }^{\mathrm{p}}$ Iontophoresis CXL treatment study group

a Scheimpflug imaging analysis (Oculus Pentacam GmbH, Wetzlar, Germany)

${ }^{\text {b }}$ Scanning slit technique (Orbscan IIz; Bausch \& LombSurgical, Rochester, NY)

and infection $(\mathrm{RD}=0.0036 ; 95 \% \mathrm{CI}-0.0206$ to 0.0279 ; $P=0.768)$. All results were obtained through fixed-effects models due to the lack of heterogeneity.

\section{Quality of the studies}

The risk of bias assessment of RCTs and NRSIs is summarized in Additional file 1. Performance bias existed in all RCTs because personnel blinding to the intervention is impossible, but the review authors judge that the outcome is not likely to be influenced by lack of blinding. There was unclear evidence of random sequence generation in two studies $[50,57]$ and high risk of bias in two other studies $[49,55]$. The allocation concealment was unclear in two studies $[49,50]$. A high risk of bias due to incomplete data was present in one study [50]. Three studies had high risk of bias [54, 55, 59] and two had unclear risk of bias $[49,60]$ in selective reporting. Overall, only two studies $[48,61]$ were of high quality with a low risk of bias, whereas two $[57,60]$ were of unclear risk of bias and five $[49,50,54,55,59]$ were of low quality with a serious risk of bias.

All the NRSIs were at serious risk of bias due to confounding factors $[47,51-53,56,58]$; moreover, Eraslan et al. [56] had a serious risk of bias in the selection of participants, Rossi et al. [51] had a serious risk of bias due to missing data and Jouve et al. [52] had a medium risk of bias in measurements of outcomes.

\section{Discussion}

CXL is now widely used to prevent the progression of keratoconus by strengthening the biomechanics of the human corneal stroma. Since its development, several clinical studies have reported the effectiveness of CXL using different transepithelial protocols and have compared the efficacy of both treatments. This meta-analysis of RCTs and NRSIs aimed at investigating the outcomes comparing different epi-on CXL protocols with standard epi-on CXL, whereas the difference in the epi-on CXL protocols used by the various authors has led to the lack of definitive evidence as to which technique is preferable.

Fifteen studies with 1085 eyes were included in the qualitative synthesis and a total of 4 studies with 264 eyes were included in the quantitative synthesis. Because the key treatment objective is to stabilize the underlying disease process, corneal topography (Kmax) was considered one of the primary outcome measures. Based on our meta-analysis, Kmax decreased slightly more after the epi-off CXL procedure but did not reach a statistically significant difference compared with epion CXL. Although CXL treatment is not intended to improve visual acuity, the induced changes in corneal topography may result in such a consequent improvement. Based on our systematic review and meta-analysis, the impact of CXL on visual acuity is remarkably different. CDVA showed a significant improvement with epi-on CXL ( $M D=0.07 ; 95 \%$ CI0.04 to 0.10$)$. Based on the MD, patients treated with epi-on CXL had a larger 0.07 logMAR CDVA improvement as compared with control subjects; nevertheless UDVA was not different between both techniques. We assumed that patients treated with CXL protocols that preserved epithelium, probably based on the fewer numbers of postoperative corneal haze, had a higher improvement of CDVA [61]. In our study, we also found that ECD and CCT had no significant changes at long-term follow-up.

A previous systematic review and meta-analysis in 2018 [62] investigated the effectiveness of conventional CXL and transepithelial CXL based on RCTs and revealed no 
Table 4 Sensitivity analysis (mean differences) for the primary and secondary outcomes in randomized clinical trials assessing corneal collagen cross-linking (epi-on vs. epi-off)

\begin{tabular}{llll}
\hline Subgroup & MD & $\mathbf{9 5 \% ~ C l}$ & $\boldsymbol{P}$ \\
\hline Kmax flattening (D) & & & \\
Low risk of bias & -1.69 & $-2.62--0.76$ & $0.004^{*}$ \\
With iontophoresis & -0.50 & $-3.67-2.67$ & 0.757 \\
UDVA (logMAR) & & & \\
Low risk of bias & -0.05 & $-0.20-0.10$ & 0.491 \\
With iontophoresis & -0.01 & $-0.23-0.21$ & 0.930 \\
CDVA (logMAR) & & & \\
Low risk of bias & 0.06 & $-0.004-0.118$ & 0.069 \\
With iontophoresis & 0.05 & $-0.01-0.11$ & 0.127 \\
CCT ( $\mu$ m) & & & \\
Low risk of bias & -2.0 & $-18.38-14.38$ & 0.811 \\
With iontophoresis & -4.76 & $-14.53-5.02$ & 0.340 \\
ECD (cells/mm ${ }^{2}$ ) & & & \\
Low risk of bias & -48.82 & $-180.79-83.15$ & 0.468 \\
With iontophoresis & 3.0 & $-22.86-28.86$ & 0.820 \\
\hline CCT centrlconea & &
\end{tabular}

CCT central corneal thickness; CDVA corrected distance visual acuity; $\mathrm{Cl}$ confidence interval; $D$ diopter; $E C D$ endothelial cell density; logMAR logarithm of the minimum angle of resolution; MD mean differences; UDVA uncorrected distance visual acuity

All the results were obtained through fixed-effects models because we only had two studies to summarize. If the value of the summary effect is negative, epi-on corneal collagen cross-linking would have more mean differences. Otherwise, epi-off would have this condition

*indicates statistical significance

significant differences from the pooled results for the UDVA, CDVA, K-steepest, or ECD. Similarly, Wen et al. [63] compared standard epithelium-off CXL and transepithelial CXL for treating keratoconus and showed no differences in UDVA, CDVA and Kmax outcomes at 1 year. However, neither of the two systematic reviews investigated the RD for adverse events related to the procedures as well as the treatment failure rate, and we are unaware of any other similar analysis in previous systematic reviews. Based on our analysis, a significant delay in epithelial healing and persistent stromal haze were found in epi-off CXL if compared to epi-on CXL (Table 5).

The standard epi-off CXL procedure in all included studies followed the Dresden protocol (central corneal epithelium was removed, riboflavin drops were instilled for $30 \mathrm{~min}$, and eyes were irradiated with UVA for $30 \mathrm{~min}$ at an irradiance of $3 \mathrm{~mW} / \mathrm{cm}^{2}$ ).

We found that the methodology of the epi-on CXL protocol varied among the included studies. To increase epithelial permeability, Al Fayez et al. [59] used benzalkonium chloride and tetracaine; Soeters et al. [61] and Rossi et al. $[51,60]$ used Ricrolin TE solution (SOOFT Italia); Al Zubi et al. [49] used $0.1 \%$ riboflavin in $20 \%$ dextran for $30 \mathrm{~min}$; Iqbal et al. [50], Henriquez et al. [53], Rush et al. [55] and Eraslan et al. [56] used benzalkonium chloride and hydroxypropyl methylcellulose. Bikbova et al. [57] performed riboflavin soaking using an iontophoresis device with $1.0 \mathrm{~mA} / \mathrm{cm}^{2}$ current density for $10 \mathrm{~min}$ to induce absorption. Lombardo et al. preceded iontophoresis by removal of the precorneal mucin layer with the intent to increase epithelial permeability to riboflavin [48]. An iontophoresis device was also applied by Vinciguerra et al. [47, 58], Rossi et al. [51, 60] and Jouve et al. [52]. However, the total energy density was equal among studies. The disagreement between the epi-on CXL protocols used in the different RCTs has led to a lack of definitive evidence which can be overcome by the scientific methodology applied in the current meta-analysis.

Studies conducted by Vinciguerra et al. [47, 58], Lombardo et al. [48] and Jouve et al. [52] used $10 \mathrm{~mW} / \mathrm{cm}^{2}$ irradiation for $9 \mathrm{~min}$, while the study conducted by $\mathrm{Al}$ Zubi et al. [49], Rossi et al. [51, 60], Rush et al. [55], Eraslan et al. [56], Bikbova et al. [57], Al Fayez et al. [59] and Soeters et al. [61] used a $3 \mathrm{~mW} / \mathrm{cm}^{2}$ irradiation device for $30 \mathrm{~min}$. Henriquez et al. [53] used an accelerated protocol to shorten $5 \mathrm{~min}$ of irradiation $\left(18 \mathrm{~mW} / \mathrm{cm}^{2}\right)$, while Iqbal et al. [50] used a pulsed protocol of irradiation (UVA $45 \mathrm{~mW} / \mathrm{cm}^{2}$ for 5:20 $\mathrm{min}$ ). Therefore, the total irradiation dose was approximately $5.4 \mathrm{~J} / \mathrm{cm}^{2}$ in each included study. The RCTs included in this study used the same irradiation energy-making this parameter homogeneous among all of them-and were compared according to whether the corneal epithelium was preserved or not.

Three of the 15 studies included in the current review treated pediatric patients with progressive keratoconus. Nevertheless, all the 4 RCTs included in the quantitative meta-analysis treated adult patients, and thus the results were not influenced by the patients' age.

The natural course of keratoconus can be long-lasting, with years of apparent stable keratometry readings after a period of latent progression. Only RCTs trials with adequate follow-up of at least one year have been included in this systematic analysis. Nevertheless, all the analyzed studies followed patients for less than 5 years. Consequently, questions about the long-term stability and efficacy of epi-on CXL compared to epi-off CXL beyond that period must be answered and RCTs comparing the long-term outcomes between these techniques are warranted.

Our study has several strengths. To the best of our knowledge, this is the first meta-analysis investigating the adverse events and treatment failure of conventional and transepithelial CXL for patients with keratoconus. Second, we update our review if compared to the previous systematic reviews on the topic: by including 3 RCTs and 1 NRSI published in 2019, we report the most 


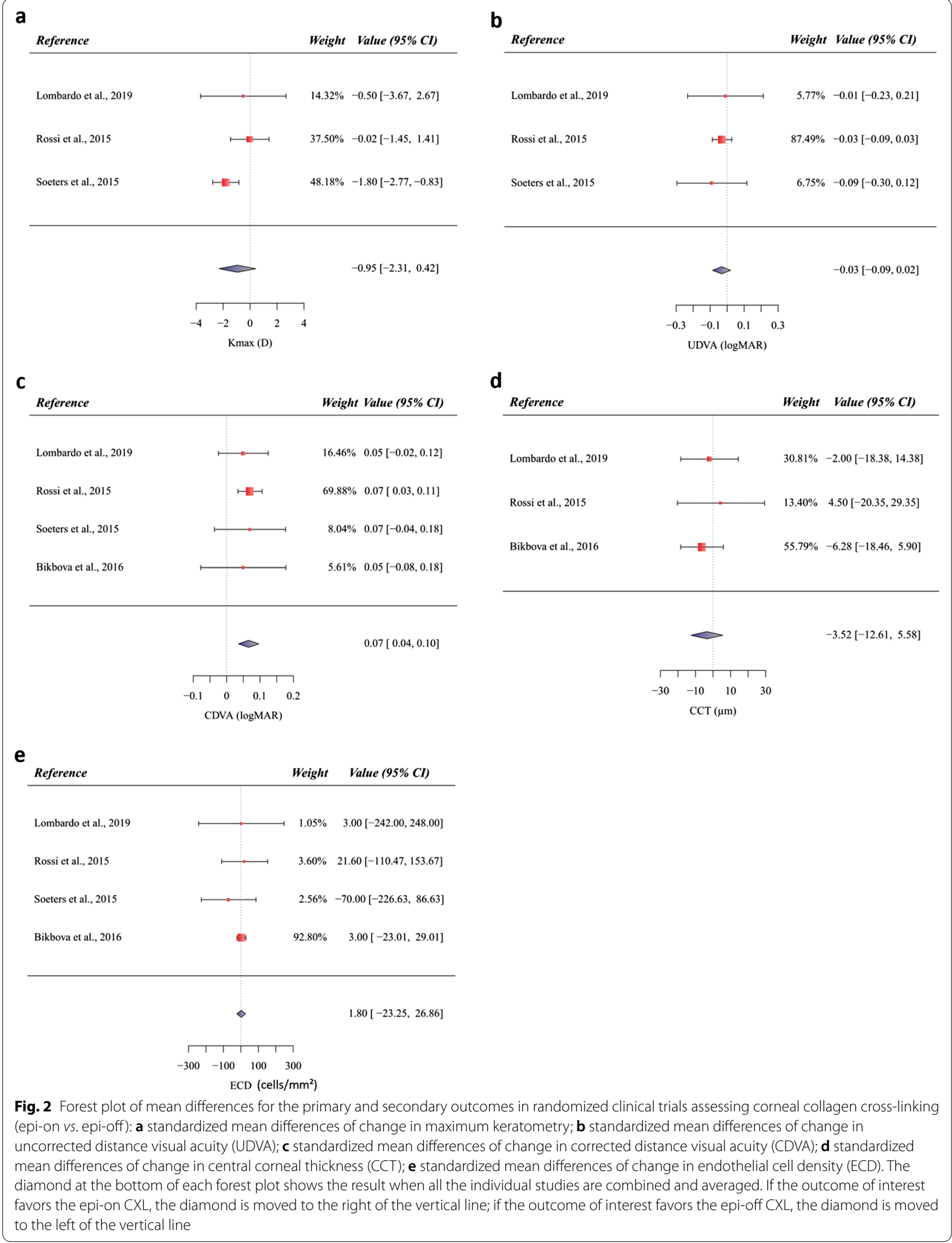


Table 5 Risk differences for the adverse effects and treatment failures in randomized clinical trials assessing corneal collagen cross-linking (epi-on vs. epi-off)

\begin{tabular}{llll}
\hline Adverse effect & RD & $\mathbf{9 5 \% ~ C l}$ & $\boldsymbol{P}$ \\
\hline Keratoconus progression & -0.025 & $-0.059-0.008$ & 0.141 \\
Delay in epithelial healing & 0.049 & $0.0030-0.0946$ & $0.035^{*}$ \\
Persistent stromal haze & 0.0525 & $0.0063-0.0986$ & $0.026^{*}$ \\
Sterile infiltrates & 0.0036 & $-0.0206-0.0279$ & 0.768 \\
Infection & 0.0036 & $-0.0206-0.0279$ & 0.768 \\
\hline
\end{tabular}

$C l$ confidence interval; $R D$ risk difference

If the value of the summary effect is negative, epi-on corneal collagen crosslinking would have more risk of adverse effects. Otherwise, epi-off would have this condition. All the results were obtained through fixed-effects models due to the lack of heterogeneity

*Indicates statistical significance

updated evidence. Third, our quantitative evaluation was based only on prospective well-designed RCTs studies with no high risk of bias, increasing the consistency of the results.

This systematic review also has several limitations that should be considered. First, like any meta-analysis, there is variability between the methodologies used for epi-on CXL among studies. However, our sensitivity analysis did not significantly alter the results when these trials using transepithelial or iontophoresis techniques were excluded. Second, we acknowledge the small number of cases per trial however, the total number of cases included in the meta-analysis gives these analyses high power. Moreover, among the 9 RCTs identified, three were published in open access journals that may or may not have limited the veracity of the data but these were not included in the meta-analysis thereby minimizing their influence on the results presented.

\section{Conclusion}

In conclusion, our systematic review and meta-analysis of the literature documenting the comparative efficacy of epi-on vs. epi-off CXL in halting the progression of keratoconus found that both protocols are equally effective. According to the studies included in the quantitative meta-analysis, the rate of keratoconus progression is not significantly different with the two protocols. The published evidence indicates that, except for a larger significant improvement in CDVA with current epi-on CXL protocols, the analysis of well-designed comparative studies available demonstrates that epi-on and epioff CXL have comparable effects on visual, topographic, pachymetric, and endothelial parameters at 1-2 years after surgery in adult patients. Epi-on CXL protocols, by preserving the epithelium, offers a significant reduction in epithelial healing delay and stromal haze formation and a faster postoperative recovery. Current evidence leaves the field open for final, confirmatory, well-designed unbiased RCTs to confirm the long-term efficacy and safety outcomes as well as similarities and differences between the epi-on and epi-off CXL techniques.

\section{Abbreviations \\ CCT: Central corneal thickness; Cl: Confidence interval; CXL: Corneal collagen cross-linking; CDVA: Corrected distance visual acuity; ECD: Endothelial cell density; HOA: Higher-order aberration; MD: Mean difference; NRSI: Non-ran- domized studies of interventions; RCT: Randomized controlled trials; RD: Risk difference; UDVA: Uncorrected distance visual acuity.}

\section{Supplementary Information}

The online version contains supplementary material available at https://doi. org/10.1186/s40662-021-00256-0.

Additional file 1. Quality evaluation and bias assessment of the included studies. a Quality evaluation and bias assessment of randomized controlled trials; $\mathbf{b}$ Quality evaluation and bias assessment of non-randomized studies of interventions.

Additional file 2. Search terms used in the MEDLINE/PubMed database and the EMBASE database.

\section{Acknowledgements}

Not applicable.

\section{Authors' contributions}

FD participated in the design of the study and data collection, drafted and revised the manuscript. AP performed the meta-analysis and revised the manuscript. JLA conceived the study and its design and participated in data collection as well as manuscript revision. All authors read and approved the final version of the manuscript.

\section{Funding}

This study has been financed in part by the Network for Cooperative Research in Health "OFTARED"-Reference: RD16/0008/0012. Funded by Instituto de Salud Carlos III and co-funded by the European Regional Development Fund (ERDF), Project "A way to make Europe".

Availability of data and materials

All data analyzed during this study are included in this published article and its Additional files.

\section{Declarations}

Ethics approval and consent to participate Not applicable.

Consent for publication

Not applicable.

\section{Competing interests}

Author Jorge L. Alio is a member of the Editorial Board for Eye and Vision. The other authors declare they have no competing interests.

\section{Author details}

${ }^{1}$ Vissum Innovation, c/ Cabañal, 1, 03016 Alicante, Spain. ${ }^{2}$ Division of Ophthalmology, Universidad Miguel Hernández, Alicante, Spain. ${ }^{3}$ Section of Ophthalmology, Department of Basic Medical Science, Neuroscience and Sense 
Organs, University of Bari, Bari, Italy. ${ }^{4}$ Department of Clinical Medicine, Migue Hernández University, San Juan de Alicante, Spain.

Received: 27 November 2020 Accepted: 8 August 2021

Published online: 26 September 2021

\section{References}

1. Arora R, Lohchab M. Pediatric keratoconus misdiagnosed as meridional amblyopia. Indian J Ophthalmol. 2019;67(4):551-2.

2. D'Oria F, Abdelghany A, Ledo N, Barraquer Rl, Alio JL. Incidence and reason for intrastromal corneal ring segment explantation. Am J Ophthalmol. 2021:222:351-8.

3. Amsler M. Kératocõne classique et kératocône fruste; arguments unitaires. Ophthalmologica. 1946;111:96-101.

4. Alió JL, Shabayek MH. Corneal higher order aberrations: a method to grade keratoconus. J refract surg. 2006;22(6):539-45.

5. Alió JL, Piñero DP, Alesón A, Teus MA, Barraquer RI, Murta J, et al. Keratoconus-integrated characterization considering anterior corneal aberrations, internal astigmatism, and corneal biomechanics. J Cataract Refract Surg. 2011;37(3):552-68.

6. Wollensak G, Sporl E, Seiler T. Treatment of keratoconus by collagen cross linking. Ophthalmologe. 2003;100(1):44-9.

7. Wollensak G, Spoerl E, Seiler T. Riboflavin/ultraviolet-A-induced collagen crosslinking for the treatment of keratoconus. Am J Ophthalmol. 2003:135(5):620-7.

8. Ghanem VC, Ghanem RC, de Oliveira R. Postoperative pain after corneal collagen cross-linking. Cornea. 2013;32(1):20-4

9. D'Oria F, Puzo P, Incandela C, Sborgia A, Boscia F, Alessio G. Evaluation of demarcation line after epithelium off iontophoresis corneal collagen cross-linking for progressive keratoconus. J Clin Med. 2021;10(13):2841.

10. Cumpston M, Li T, Page MJ, Chandler J, Welch VA, Higgins JP, et al. Updated guidance for trusted systematic reviews: a new edition of the Cochrane Handbook for Systematic Reviews of Interventions. Cochrane Database Syst Rev. 2019:10:ED000142.

11. Moher D, Liberati A, Tetzlaff J, Altman DG, PRISMA Group. Preferred reporting items for systematic reviews and meta-analyses: the PRISMA statement. PLoS Med. 2009;6(7):e1000097.

12. Shrier I, Boivin JF, Steele RJ, Platt RW, Furlan A, Kakuma R, et al. Should meta-analyses of interventions include observational studies in addition to randomized controlled trials? A critical examination of underlying principles. Am J Epidemiol. 2007;166(10):1203-9.

13. World Health Organization. General guidelines for methodologies on research and evaluation of traditional medicine. Geneva: World Health Organization; 2000

14. Sterne JA, Hernan MA, Reeves BC, Savović J, Berkman ND, Viswanathan M et al. ROBINS-I: a tool for assessing risk of bias in non-randomised studies of interventions. BMJ. 2016:355:i4919.

15. Shea BJ, Reeves BC, Wells G, Thuku M, Hamel C, Moran J, et al. AMSTAR 2: a critical appraisal tool for systematic reviews that include randomised or non-randomised studies of healthcare interventions, or both. BMJ. 2017;358:j4008.

16. Higgins J, Thompson S, Deeks J, Altman D. Statistical heterogeneity in systematic reviews of clinical trials: a critical appraisal of guidelines and practice. J Health Serv Res Policy. 2002;7(1):51-61.

17. Sterne JA, Sutton AJ, loannidis JP, Terrin N, Jones DR, Lau J, et al. Recommendations for examining and interpreting funnel plot asymmetry in meta-analyses of randomised controlled trials. BMJ. 2011;343:d4002.

18. Abdelmassih Y, El-Khoury S, Dirani A, Antonios R, Fadlallah A, Cherfan $C G$, et al. Safety and efficacy of sequential intracorneal ring segment implantation and cross-linking in pediatric keratoconus. Am J Ophthalmol. 2017;178:51-7.

19. Choi M, Kim J, Kim EK, Seo KY, Kim TI. Comparison of the conventional Dresden protocol and accelerated protocol with higher ultraviolet intensity in corneal collagen cross-linking for keratoconus. Cornea. 2017:36(5):523-9.

20. Cummings AB, McQuaid R, Naughton S, Brennan E, Mrochen M. Optimizing corneal cross-linking in the treatment of keratoconus: a comparison of outcomes after standard- and high-intensity protocols. Cornea. 2016:35(6):814-22.
21. Eissa SA, Yassin A. Prospective, randomized contralateral eye study of accelerated and conventional corneal cross-linking in pediatric keratoconus. Int Ophthalmol. 2019;39(5):971-9.

22. Godefrooij DA, Roohé SL, Soeters N, Wisse RPL. The independent effect of various cross-linking treatment modalities on treatment effectiveness in keratoconus. Cornea. 2020;39(1):63-70.

23. Hashemian $H$, Jabbarvand $M$, Khodaparast M, Ameli K. Evaluation of corneal changes after conventional versus accelerated corneal cross-linking: a randomized controlled trial. J Refract surg. 2014;30(12):837-42.

24. Hashemi H, Miraftab M, Seyedian MA, Hafezi F, Bahrmandy H, Heidarian S, et al. Long-term results of an accelerated corneal cross-linking protocol $\left(18 \mathrm{~mW} / \mathrm{cm}^{2}\right)$ for the treatment of progressive keratoconus. Am J Ophthalmol. 2015;160(6):1164-70.

25. Kato N, Konomi K, Shinzawa M, Kasai K, Ide T, Toda I, et al. Corneal crosslinking for keratoconus in Japanese populations: one year outcomes and a comparison between conventional and accelerated procedures. Jpn J Ophthalmol. 2018;62(5):560-7

26. Knyazer B, Kormas RM, Chorny A, Lifshitz T, Achiron A, Mimouni M Corneal cross-linking in thin corneas: 1-year results of accelerated contact lens-assisted treatment of keratoconus. J Refract surg. 2019;35(10):642-8.

27. Ng AL, Chan TC, Cheng AC. Conventional versus accelerated corneal collagen cross-linking in the treatment of keratoconus. Clin Exp Ophthalmol. 2016:44(1):8-14.

28. Sadoughi MM, Einollahi B, Baradaran-Rafii A, Roshandel D, Hasani H, Nazeri M. Accelerated versus conventional corneal collagen cross-linking in patients with keratoconus: an intrapatient comparative study. Int Ophthalmol. 2018;38(1):67-74.

29. Seiler TG, Fischinger I, Koller T, Zapp D, Frueh BE, Seiler T, et al. Customized corneal cross-linking: one-year results. Am J Ophthalmol. 2016;166:14-21.

30. Tomita M, Mita M, Huseynova T. Accelerated versus conventional corneal collagen crosslinking. J Cataract Refract Surg. 2014:40(60):1013-20.

31. Bilgihan K, Yesilirmak N, Altay Y, Yuvarlak A, Ozdemir HB. Conventional corneal collagen cross-linking versus transepithelial diluted alcohol and ontophoresis-assisted corneal cross-linking in progressive keratoconus. Cornea. 2017;36(12):1492-7.

32. Buzzonetti L, Petrocelli G, Valente P, larossi G, Ardia R, Petroni S, et al. Iontophoretic transepithelial collagen cross-linking versus epitheliumoff collagen cross-linking in pediatric patients: 3-year follow-up. Cornea. 2019;38(7):859-63.

33. Cantemir A, Alexa Al, Anton N, Ciuntu RE, Danielescu C, Chiselita D, et al. Evaluation of iontophoretic collagen cross-linking for early stage of progressive keratoconus compared to standard cross-linking: a noninferiority study. Ophthalmol Ther. 2017;6(1):147-60.

34. Cantemir A, Alexa Al, Galan BG, Anton N, Ciuntu RE, Danielescu C, et al. lontophoretic collagen cross-linking versus epithelium-off collagen crosslinking for early stage of progressive keratoconus - 3 years follow-up study. Acta Ophthalmol. 2017;95(7):e649-55.

35. Çerman EE, Toker E, Ozarslan OD. Transepithelial versus epithelium-off crosslinking in adults with progressive keratoconus. J Cataract Refract Surg. 2015;41(7):1416-25.

36. Madeira C, Vasques A, Beato J, Godinho G, Torrão L, Falcão M, et al. Transepithelial accelerated versus conventional corneal collagen crosslinking in patients with keratoconus: a comparative study. Clin Ophthalmol. 2019;13:445-52.

37. Sarac O, Caglayan M, Uysal BS, Uzel AGT, Tanriverdi B, Cagil N. Accelerated versus standard corneal collagen cross-linking in pediatric keratoconus patients: 24 months follow-up results. Cont Lens Anterior eye. 2018;41(5):442-7.

38. Tian M, Jian W, Sun L, Shen Y, Zhang X, Zhou X. One-year follow-up of accelerated transepithelial corneal collagen cross-linking for progressive pediatric keratoconus. BMC Ophthalmol. 2018;18(1):75.

39. Serrao S, Lombardo G, Giannini D, Lombardo M. Corneal topography and aberrometry changes one-year after transepithelial corneal cross-linking using iontophoresis versus standard corneal cross-linking. Invest Ophthalmol Vis Sci. 2017;58(8):3504.

40. Medina RB, Ramirez-Miranda AJ, Graue-Hernandez EO, Cabral J, EscuderoRodriguez A. Efficacy, safety, clinical and topographic outcomes of two corneal crosslinking for the treatment keratoconus in a referral center. Invest Ophthalmol Vis Sci. 2017:58(8):3522.

41. Munoz JAS, Gonzalez Mendoza EA, Ramirez-Miranda AJ, Navas A, Graue-Hernandez EO. Comparison of corneal topographic characteristics 
between eyes with indication of transepithelial (epi-on) and epi-off cross linking for treatment of keratoconus in a reference center in Mexico City. Invest Ophthalmol Vis Sci. 2018;59(8):4384.

42. Godefrooij DA, Roohé SL, Soeters N, Wisse RPL. The independent effect of various cross-linking treatment modalities on treatment effectiveness in keratoconus. Cornea. 2020;39(1):63-70.

43. Atia R, Jouve L, Sandali O, Laroche L, Borderie V, Bouheraoua N. Early epithelial remodeling after standard and iontophoresis-assisted corneal cross-linking as evaluated by spectral-domain optical coherence tomography. J Refract surg. 2018;34(8):551-8.

44. Cassagne M, Laurent C, Rodrigues M, Galinier A, Spoerl E, Galiacy SD, et al. Iontophoresis transcorneal delivery technique for transepithelial corneal collagen crosslinking with riboflavin in a rabbit model. Invest Ophthalmol Vis Sci. 2016;57(2):594-603.

45. Lombardo M, Giannini D, Lombardo G, Serrao S. Randomized controlled trial comparing transepithelial corneal cross-linking using iontophoresis with the dresden protocol in progressive keratoconus. Ophthalmology. 2017;124(6):804-12

46. Yuksel E, Novruzlu S, Ozmen MC, Bilgihan K. A study comparing standard and transepithelial collagen cross-linking riboflavin solutions: epithelial findings and pain scores. J Ocul Pharmacol Ther. 2015;31(5):296-302.

47. Vinciguerra P, Rosetta P, Legrottaglie EF, Morenghi E, Mazzotta C, Kaye $\mathrm{SB}$, et al. Iontophoresis CXL with and without epithelial debridement versus standard CXL: 2-year clinical results of a prospective clinical study. J Refract Surg. 2019;35(3):184-90.

48. Lombardo M, Serrao S, Lombardo G, Schiano-Lomoriello D. Two-year outcomes of a randomized controlled trial of transepithelial corneal crosslinking with iontophoresis for keratoconus. J Cataract Refract Surg. 2019;45(7):992-1000.

49. Al Zubi KAY, Nasser R. Transepithelial versus epithelium off crosslinking for treating keratoconus among Jordanians. Open Ophthalmol J. 2019;13:8-14.

50. Iqbal M, Elmassry A, Saad H, Am Gad A, Ibrahim O, Hamed N, et al. Standard cross-linking protocol versus accelerated and transepithelial cross-linking protocols for treatment of paediatric keratoconus: a 2-year comparative study. Acta Ophthalmol. 2019;98(3):e352-62.

51. Rossi S, Santamaria C, Boccia R, De Rosa L, D'Alterio FM, Simonelli F, et al. Standard, transepithelial and iontophoresis corneal cross-linking: clinical analysis of three surgical techniques. Int Ophthalmol. 2018;38(6):2585-92.

52. Jouve L, Borderie V, Sandali O, Temstet C, Basli E, Laroche L, et al. Conventional and iontophoresis corneal cross-linking for keratoconus: efficacy and assessment by optical coherence tomography and confocal microscopy. Cornea. 2017;36(2):153-62.
53. Henriquez MA, Rodriguez AM, Izquierdo L Jr. Accelerated epi-on versus standard epi-off corneal collagen cross-linking for progressive keratoconus in pediatric patients. Cornea. 2017:36(12):1503-8.

54. Godefrooij DA, Kandoussi ME, Soeters N, Wisse RP. Higher order optical aberrations and visual acuity in a randomized controlled trial comparing transepithelial versus epithelium-off corneal crosslinking for progressive keratoconus. Clin Ophthalmol. 2017;11:1931-6. https://doi.org/10.2147/ OPTH.S139358.

55. Rush SW, Rush RB. Epithelium-off versus transepithelial corneal collagen crosslinking for progressive corneal ectasia: a randomised and controlled trial. Br J Ophthalmol. 2017;101(4):503-8.

56. Eraslan M, Toker E, Cerman E, Ozarslan D. Efficacy of epithelium-off and epithelium-on corneal collagen cross-linking in pediatric keratoconus. Eye Contact Lens. 2017;43(3):155-61.

57. Bikbova G, Bikbov M. Standard corneal collagen crosslinking versus transepithelial iontophoresis-assisted corneal crosslinking, 24 months follow-up: randomized control trial. Acta Ophthalmol. 2016;94(7):e600-6.

58. Vinciguerra P, Romano V, Rosetta P, Legrottaglie EF, Piscopo R, Fabiani $C$, et al. Transepithelial iontophoresis versus standard corneal collagen cross-linking: 1-year results of a prospective clinical study. J Refractive Surg. 2016;32(10):672-8

59. Al Fayez MF, Alfayez S, Alfayez Y. Transepithelial versus epithelium-off corneal collagen cross-linking for progressive keratoconus: a prospective randomized controlled trial. Cornea. 2015;34(Suppl 10):S53-6.

60. Rossi S, Orrico A, Santamaria C, Romano V, De Rosa L, Simonelli F, et al. Standard versus trans-epithelial collagen cross-linking in keratoconus patients suitable for standard collagen cross-linking. Clin Ophthalmol. 2015;9:503-9.

61. Soeters N, Wisse RP, Godefrooij DA, Imhof SM, Tahzib NG. Transepithelial versus epithelium-off corneal cross-linking for the treatment of progressive keratoconus: a randomized controlled trial. Am J Ophthalmol. 2015;159(5):821-8.e3.

62. Zhang $X$, Zhao J, Li M, Tian M, Shen Y, Zhou X. Conventional and transepithelial corneal cross-linking for patients with keratoconus. PLoS One. 2018;13(4):e0195105

63. Wen D, Song B, Li Q, Tu R, Huang Y, Wang Q, et al. Comparison of epithelium-off versus transepithelial corneal collagen cross-linking for keratoconus: a systematic review and meta-analysis. Cornea. 2018;37(8):1018-24.

\section{Publisher's Note}

Springer Nature remains neutral with regard to jurisdictional claims in published maps and institutional affiliations.
Ready to submit your research? Choose BMC and benefit from:

- fast, convenient online submission

- thorough peer review by experienced researchers in your field

- rapid publication on acceptance

- support for research data, including large and complex data types

- gold Open Access which fosters wider collaboration and increased citations

- maximum visibility for your research: over 100M website views per year

At BMC, research is always in progress.

Learn more biomedcentral.com/submissions 